\title{
A Particle Sampler for Trace Detection of Explosives
}

\author{
Sebastian Beer $^{1,2}$, Gerhard Müller ${ }^{1}$, Jürgen Wöllenstein ${ }^{2}$ \\ EADS Innovation Works, 81663 Munich, Germany \\ ${ }^{2}$ University of Freiburg, IMTEK, Georges-Köhler-Allee 106, 7911 Freiburg, Germany \\ Sebastian.Beer@eads.net
}

\begin{abstract}
:
We present our developments toward a handheld field-ready trace explosives detection system using an electrostatic particle sampler with an integrated thermal desorber. Particle sampling with subsequent thermal desorption is used to overcome the problem of low vapor pressure of explosives. A degree of selectivity toward high electron affinity, characteristic for most explosives, is demonstrated experimentally. This reduces the detection background and improves the system performance. Detection is shown in applications of the sampler to both commercial detectors as well as novel miniaturized sensors.
\end{abstract}

Key words: Trace explosives detection, particle sampling, electrostatic precipitation, thermal desorption, particle gas conversion.

\section{Background}

Due to terrorist attacks using explosives in a wide range of attacks [1], there is urgent need for the development of field-operable, handheld trace explosives detectors. Most explosives have a very low vapor pressure and are therefore extremely difficult to detect directly via gas detectors [2]. One approach to addressing this issue is the collection of trace particulates followed by thermal desorption for a temporarily increased gas concentration detectable by gas sensors. Reports show that explosives adsorb onto particulates [3], making this approach feasible for a detection system. We report on our development of an electrostatic sampling and integrated thermal desorption unit to be used in conjunction with miniaturized gas detectors to form a handheld explosives detection system.

\section{Design}

Fig. 1 outlines the basic sampling and desorption procedure. Collection is based on electrostatic precipitation (ESP) [4], with a large sampling air flow. A needle-plate electrode arrangement is used for the corona electrodes. Depending on the operating parameters, such as the sampling polarity and artificially added humidity, a degree of selectivity toward the high electron affinity of explosives [5] is introduced in the sampling.

Using a combined collection and heating electrode, precipitation and desorption can be performed in the same location. During desorption a small carrier gas flow is used for transport to the detector to reduce analyte dilution. An alternative procedure using sampler transfer to a separate detection chamber, without the use of a transport gas has also been implemented. Its advantage lies in reduced analyte dilution and easier cell cleaning, while its drawbacks are longer and more complicated handling and reduced suitability for automation.
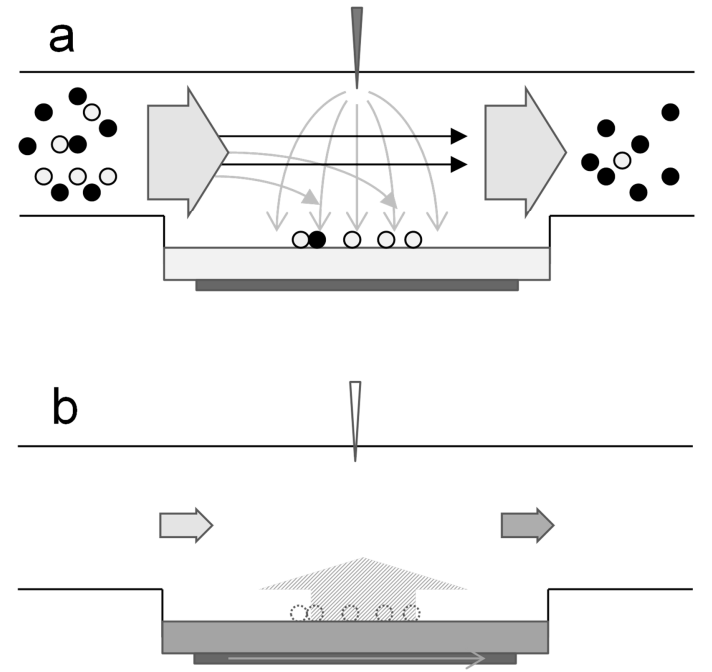

Fig. 1. Outline of the basic sampler operation: (a, top) precipitation step, with potentially interesting particles (light) collected via electrostatic precipitation while dust (dark) is discarded; (b, bottom) desorption step into a smaller carrier gas flow to the detector. 


\section{Experimental Results}

The potential for selectivity was demonstrated experimentally using a Lugol's solution, which indicates miniscule amounts of starch by turning black, as shown in Fig. 2. Sampling from a $\mathrm{TiO}_{2}$ (high electron affinity) and ground flour (low electron affinity) mixture, the solution shows that no significant amount of flour was precipitated electrostatically.

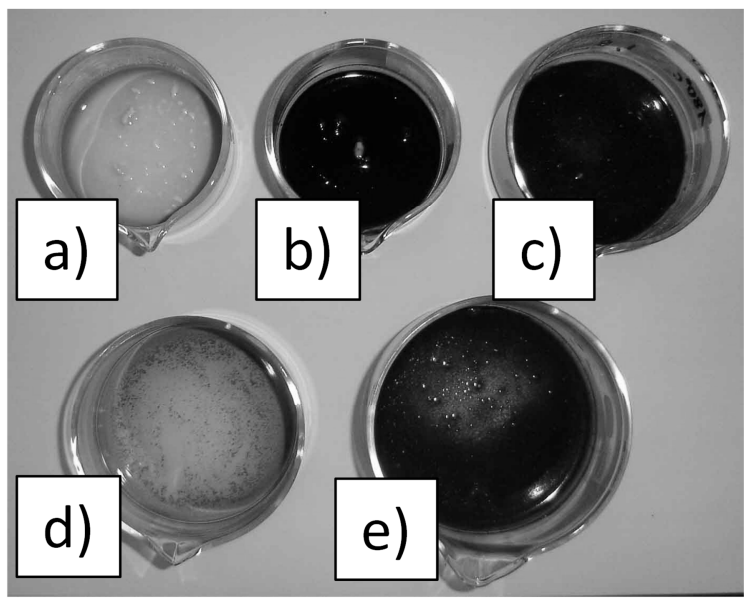

Fig. 2. Set of Lugol's solution samples, shown after particle insertion. Top row reference samples: a) pure $\mathrm{TiO}_{2}$, b) pure flour, c) $\mathrm{TiO}_{2}$ and flour mix with flour effect dominating clearly. The bottom row shows samples from a mixed $\mathrm{TiO}_{2}$ and flour precipitation experiment: d) collected particles, e) discarded particles.

The effects of performance parameters were investigated and sampler operation was characterized. In preliminary yield experiments using dinitronaphtalene (a substitute for explosives), approximately $25 \%$ of inserted particle mass was collected for smaller $(\sim 1 \mathrm{mg})$ insertion amounts. Comparing the inserted and collected size distributions, as shown in Fig. 3, shows that predominantly the smaller particles are collected.

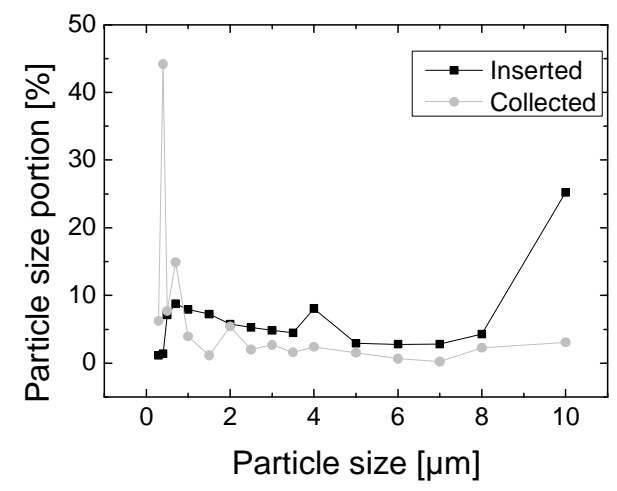

Fig. 3. Particle size distribution comparison between the inserted (dark) and collected (light) $\mathrm{TiO}_{2}$ particles, showing that a significantly larger portion of the smaller $(<1 \mu \mathrm{m})$ particles are collected.
Successful detection of ESP-sampled dinitrotoluene (DNT) particles identified via ion mobility spectrometry in a sample transfer benchmarking experiment with commercial detection hardware (Smiths Ionscan) is demonstrated.

Furthermore, the function of the integrated desorber coupled to a metal oxide gas sensor array is shown in experiments as a development toward a handheld system.

\section{References}

[1] A. Bevelacqua, R. Stilp, Terrorism Handbook for Operational Responders, 3rd Ed., Delmar (2009) ISBN-10: 1428311459

[2] B. C. Dionne et al., Vapor pressure of explosives, J. Energetic Mater. 4, 1, $447-472$ (1986); doi: $10.1080 / 07370658608011353$

[3] J. R. Verkouteren, Particle characteristics of trace high explosives: RDX and PETN, J. Forensic Sci. 52, 2, 335-340 (2007); doi: 10.1111/j.15564029.2006.00354.x

[4] J. I. Steinfeld, J. Wormhoudt, Explosives detection: a challenge for physical chemistry, Annu. Rev. Phys. Chem. 49, 203-232 (1998); doi: 10.1146/annurev.physchem.49.1.203

[5] A. Mizuno, Electrostatic precipitation, IEEE Trans. Dielect. Electr. Insul. 7, 5, 615-624 (2000); doi: 10.1109/94.879357 